\title{
Post-infectious encephalomyelitis in an orthopedic surgeon with motor and cognitive impairment following Campylobacter gastroenteritis with complete recovery
}

\author{
Christopher J Crist MD, Nattamol Hosiriluck MD, Richard E Winn MD
}

\begin{abstract}
Acute disseminated encephalomyelitis, also known as post-infectious encephalomyelitis, is an acute central nervous system demyelinating disorder which typically follows an autoimmune response secondary to a post-viral infection/syndrome. Although uncommon, the outcome can be devastating; mortality is not high but the morbidity may be catastrophic. Survival is anticipated but return to full function of highly motor skilled and cognitive individuals may not be expected. An orthopedic surgeon developed an acute autoimmune encephalitis presumed to be due to Campylobacter jejuni and despite initial significant cognitive and motor deficits was able to recover fully and ultimately return to his specialty surgical occupation.
\end{abstract}

Keywords: Acute disseminated encephalomyelitis, post-infectious encephalomyelitis, Campylobacter

\section{INTRODUCTION}

Acute disseminated encephalomyelitis (ADEM) results from immunologic responses secondary to similarities of microbial and human antigens. Both active infections and immunizations have been associated with this syndrome. Symptoms, including disorientation and lethargy, are acute and can rapidly progress within 24 hours of onset. ${ }^{1}$ Associated infections have included viral pathogens, such as Epstein-Barr virus, Herpes simplex virus, human Herpes virus-6, influenza, HIV, rubeola, rubella, mumps, varicella, measles, and smallpox, and Mycoplasma pneumoniae..$^{2-6}$ The long term consequences of ADEM can be catastrophic with persistent neurologic deficits, including

Corresponding author: Christopher Crist Contact Information: Christopher.crist@ttuhsc.edu DOI: $10.12746 /$ swrccc.v6i23.462 chronic vegetative states. Details are sparse about the types of persistent disability and the long term outlook for quality of life.

\section{Case Presentation}

A 36-year-old male orthopedic surgeon with no significant past medical history presented with lethargy and altered mental status. No health issues existed prior to a dove hunting trip 12 days prior to admission. Intermittent fever developed with multiple episodes of diarrhea lasting 10 days. When evaluated by a physician, diarrhea was confirmed, and fever recorded at $103^{\circ} \mathrm{F}$. Oral ciprofloxacin and metronidazole were started. Campylobacter jejuni was isolated from stool cultures. Erythromycin was substituted for the other antibiotics for 10 days. Recurrent fever was noted with the abrupt development of altered mental status and lethargy. His surgical schedule had continued at its usual pace throughout the febrile, diarrheal illness up to the day prior to admission. 


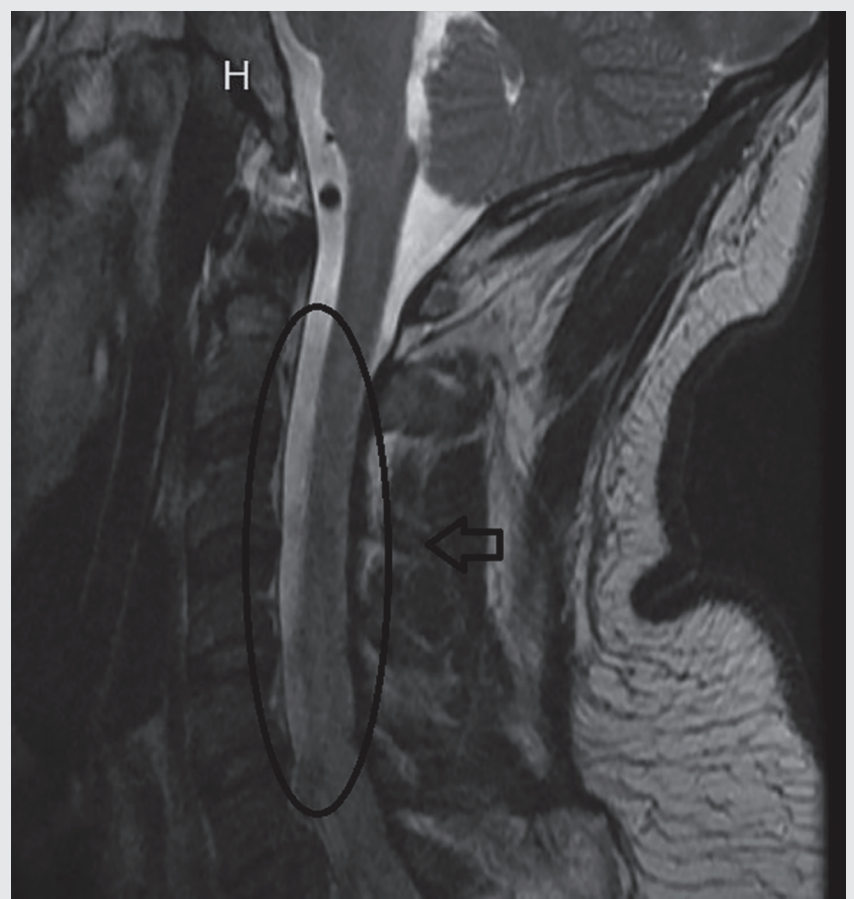

Figure 1. Patchy areas of $\mathrm{T} 2$ signal hyperintensity are noted in the cervical spinal cord.

Disorientation and lethargy were noted; responses to voice and noxious stimuli were present. Oral temperature was $102.3^{\circ} \mathrm{F}$. Pupils were small but equal and reactive to light. Extraocular movement appeared random and spontaneous but were coordinated without strabismus or ophthalmoplegia. Corneal reflexes were intact. Passive limb motion was normal, but active range of motion of limbs, including motor power, could not be assessed due to his lack of response to verbal commands. Furthermore, sensory function beyond noxious stimuli could not be tested given his mental status. Deep tendon reflexes were hyperactive with clonus; the Babinski sign was negative bilaterally. Nuchal rigidity was absent. Lorazepam was administered for observed seizure activity followed by levetiracetam and phosphenytoin. Computed tomography (CT) of the head showed no mass, midline shift, intracranial bleeding, or increased intraventricular pressure. Lumbar puncture studies revealed clear, colorless cerebral spinal fluid (CSF), RBC $71 / \mathrm{mm}^{3}$, and WBC $261 / \mathrm{mm}^{3}$ with $32 \%$ neutrophils,
$50 \%$ lymphocytes, $17 \%$ monocytes, and no eosinophils. Glucose was $59 \mathrm{mg} / \mathrm{dl}$ and protein was within the normal range. Empiric parenteral vancomycin, ceftriaxone, and acyclovir were started.

Infectious meningitis complicated by seizures was the initial diagnosis suggested by the Neurology service. The ceftriaxone dose was increased by the Infectious Diseases service. Metronidazole-induced meningoencephalitis was considered, and an EEG and magnetic resonance imaging (MRI) were ordered. The EEG done after a significant reduction in GCS score requiring intubation for airway protection demonstrated diffuse slowing but no seizure activity. Repeat CT of the head was normal. The MRI demonstrated multiple areas of T2 signal hyper-intensity in the spinal cord, dorsal pons, superior cerebellar peduncle, and middle cerebellar peduncles that were interpreted as demyelinating white matter disease. There was some paranasal sinus mucosal thickening.

Intravenous immunoglobulin G (IVIG) was given once daily for 5 days starting on day three of admission for presumptive ADEM secondary to $C$. jejuni. On day five the MRI was repeated and confirmed pons and cerebellar involvement with increased T2 signal intensity extending into the corpus callosum bilaterally. Parenteral methylprednisolone was started. No significant improvement occurred, and he remained in coma. Fever had abated, the bacterial antigen panel and cultures of CSF were negative, and vancomycin and ceftriaxone were discontinued. Doxycycline was begun, and acyclovir was continued. Six days after admission responses to loud verbal stimuli and some delayed ocular tracking were observed. Herpes simplex virus PCR of CSF was negative; acyclovir was discontinued. A five-day course of high dose IV methylprednisolone was completed.

Serologic tests for other infectious agents were all negative; doxycycline and ampicillin were discontinued. Antinuclear antibody and anti-SSA antibody (1.3 U) were elevated; anti-SSB was negative. At day 12 , prednisone was initiated with a taper over a few weeks. He slowly improved from initial limited response to simple commands to appropriate but delayed response without verbal ability. At the time of transfer (weeks) more complex commands were 


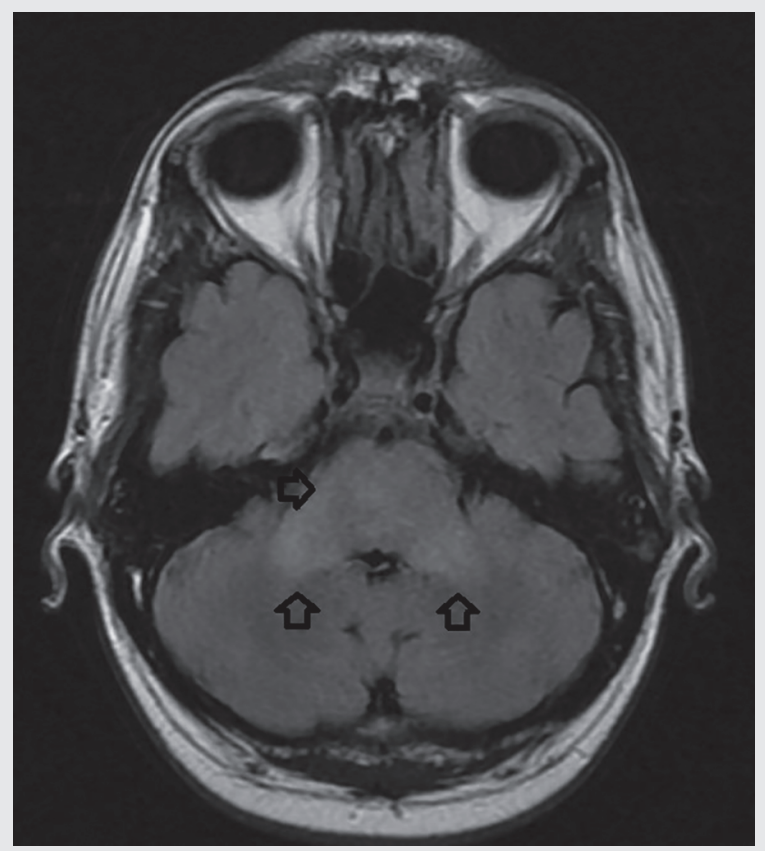

Figure 2. T2 signal hyper-intensity is noted diffusely in the pons as well as the bilateral middle cerebellar peduncles.

followed accompanied by attempts at verbalization and improved response times. The MRI still revealed cerebellar involvement (Figure 2). Loss of verbal inhibition and insight was present, especially with his orthopedic colleagues; this behavior improved when he became more awake and responsive. This was attributed to involvement of the right frontal-parietal area of the brain (Figure 3). On day 20 he was transferred to a neurologic specialty inpatient rehabilitation center in Houston, TX.

Serum gangliosidase GM1 IgM was elevated at $1: 1600$ with IgG $<1: 800$, indicating an associated autoimmune inflammatory response. Acute disseminated encephalomyelitis was confirmed; progressive clinical improvement occurred despite persistent radiographic abnormalities. His clinical evolution was consistent with published observations associating Ganglioside GM1 IgM elevation with ADEM followed by gradual clinical improvement after IVIG/plasmapheresis and corticosteroid treatment. ${ }^{7}$

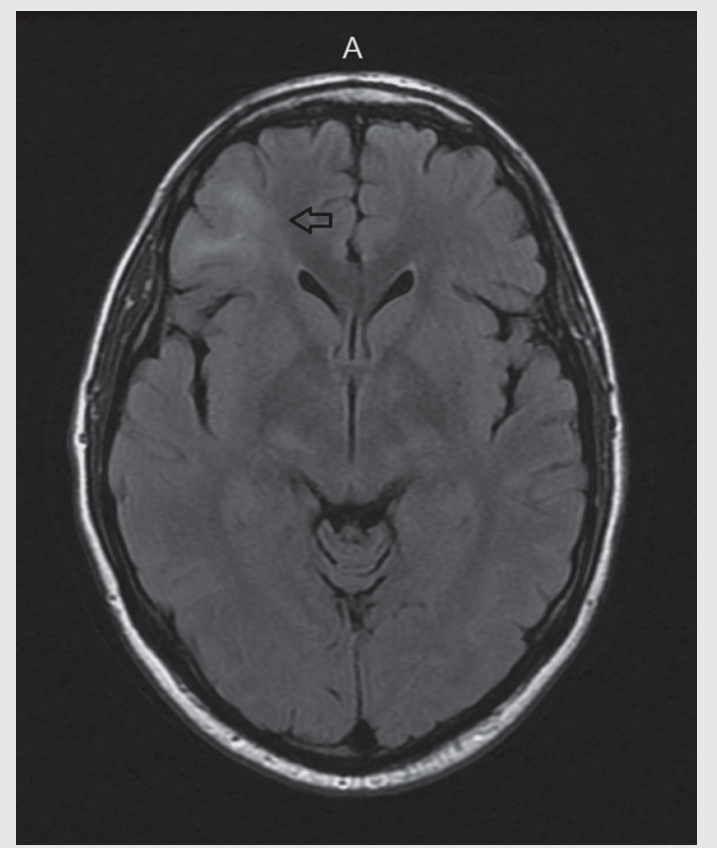

Figure 3. Area of T2 signal hyper-intensity is noted in the right frontal lobe.

Intensive neurologic rehabilitation improved his mental and motor function back to his premorbid baseline; urinary incontinence lasted three months after discharge. At six months he returned to work in a limited capacity with significant supervisory restriction; he returned to full working capacity as an orthopedic surgeon within one year from initial hospitalization. He has had no documented relapse or recurrence since the initial disease over three years ago and continues to work currently at full capacity as an orthopedic surgeon.

\section{Discussion}

Acute disseminated encephalomyelitis typically follows an immunologic response secondary to a postviral infection/syndrome. One proposed mechanism for its pathogenesis involves myelin autoantigens that are similar to the recent infective antigens, resulting in a cell-mediated response cross reacting with the autoantigens that lead to acute rapid demyelination 
causing CNS symptoms. ${ }^{1}$ Another proposed mechanism suggests that ADEM may be secondary to elevation in vascular permeability and subsequent congestion of the CNS due to an increase in immune complexes in circulation influenced by introduction of infectious or vaccination associated antigens. This process would likely result in vessel wall infiltration by mononuclear cells resulting in perivenous edema and possibly hemorrhages; infiltrating mononuclear cells and microglia could cause demyelination, gliosis, and potentially necrosis. $^{8}$

Acute disseminated encephalomyelitis is relatively uncommon, and the risk after infection is unknown. Seroprevalence surveys have suggested that immunologic reactivity is higher with Campylobacter than other pathogens, including Yersinia. ${ }^{9}$ In a meta-analysis of Campylobacter complications, the risk of GuillainBarre syndrome was reported to be $0.07 \%$, while the risks of developing reactive arthritis was $2.86 \%$, and irritable bowel syndrome was $4.01 \%{ }^{10}$

Previous reports describing post-infectious encephalomyelitis following Campylobacter enteric infections with immunologic sequelae have documented progressive improvement to normal function despite radiographic evidence showing acute demyelinating disease. ${ }^{11-12}$ Orr, et al. reported a 24-year-old man with headache, fever, and night sweats with a four-day history of diarrhea and a positive stool culture for Campylobacter sp. Two days later, disorientation, slurred speech, diplopia, and gait disturbance developed. An elevated WBC $\left(541 / \mathrm{mm}^{3}\right)$ in the CSF, slow wave activity on EEG, and T2 MRI imaging showing "multiple high signal foci in the supra and infra-tentorial compartments involving the cortex, white matter, and deep gray matter" were described. Improvement was noted following administration of methylprednisolone with full recovery in six weeks following the onset. ${ }^{12}$ Other authors have reported patients with some residual neurologic dysfunction. ${ }^{13-15}$ Gaig, et al. reported a 38-year-old woman with CNS symptoms following a flu-like syndrome with diarrhea two weeks prior to the onset of symptoms and no recent history of vaccination. She had "hyper-intense lesions on T2 weighted images in the dorsal pons extending into the medulla and the dorsal cord between T6 and T11 without gadolinium enhancement" on MRI. Antiganglioside GM1 antibodies were positive with a high titer. In addition, she had initial symptoms of neurogenic bladder which lasted five months. No recurrence was described. ${ }^{13}$ Young, et al. documented a 20-year-old woman who developed profound lethargy with reduced level of consciousness, progressive generalized weakness, and urinary retention occurring four days following an eight-day acute illness with fever, bloody diarrhea, and vomiting. Cranial and spinal MRIs demonstrated "extensive confluent high signal in the dorsal aspect of the pons extending into the base of the left middle cerebellar peduncle as well as the cervical and thoracic cord." Methylprednisolone treatment was unsuccessful; however, improvement was seen after plasmapheresis. Persistent urinary urgency was the only residual symptom (46 days). Serum antiganglioside antibody titers performed before plasmapheresis demonstrated high elevation of IgM antibody titer. ${ }^{16}$ None of these patients had a clinical recurrence/ relapse.

Our patient's clinical presentation and complete recovery are consistent with prior reports associating ganglioside GM1 IgM elevation with ADEM and Campylobacter induced disease. Intravenous immunoglobulin $\mathrm{G}$ and/or plasmapheresis and corticosteroids have all been used in previously reported cases. A favorable outcome, however, is unusual for antibody mediated immunologic encephalomyelitis. ${ }^{17-22}$ To our knowledge, this is the first known reported occurrence of Campylobacter associated ADEM in the United States and documents the return of full cognitive and motor function in a surgeon.

\section{Conclusion}

The case demonstrates the importance of recognizing a rare acute CNS demyelinating disorder with encephalopathy preceded by a non-CNS infection. In this instance, Campylobacter associated gastroenteritis was the preceding infectious process. Although the clinical features of ADEM can mimic a central nervous system infection, such as encephalitis or meningitis, it is crucial to consider a post-infectious immunologic etiology as part of the differential diagnosis. Early and accurate clinical assessment of ADEM utilizing key clinical and radiographic features in a case of acute 
encephalitis can direct further workup to include lab studies such as serum gangliosidase GM1 IgM to support diagnosis, as well as avoid unnecessary use of antimicrobial agents in this setting after this alternative diagnosis is highly suspected or established.

Specific details of cognitive and motor improvement after ADEM associated with Campylobacter and other antecedent infectious agents are limited and mostly report a return to basal activities of daily living. This case emphasizes that higher cognitive and motor function can occur despite severe CNS functional impairment when comprehensive medical care is combined with intensive rehabilitation. Intravenous immunoglobulin $\mathrm{G}$ and/or plasmapheresis with corticosteroids may contribute to successful outcomes. The return of high level cognition and fine motor control may take months or perhaps years but is possible. It does, however, appear prudent to objectively assess occupational skills requiring significant motor and cognitive skills after recovery to ensure safety.

\section{KEY STATEMENTS}

1. This case illustrates the potential for full functional recovery in patients with ADEM.

2. Future studies should record the frequency of neurological symptoms and neurological syndromes after episodes of Campylobacter diarrhea.

Article citation: Crist CJ, Hosiriluck N, Winn RE. Post-infectious encephalomyelitis in an orthopedic surgeon with motor and cognitive impairment following Campylobacter gastroenteritis with complete recovery. The Southwest Respiratory and Critical Care Chronicles 2018;6(23):22-27

From: The Department of Internal Medicine at Texas Tech University Health Sciences Center, Lubbock, TX Submitted: $12 / 5 / 2017$

Accepted: 4/4/2018

Reviewer: Doungporn Ruthirago MD

Conflicts of interest: none

This work is licensed under a Creative Commons Attribution-ShareAlike 4.0 International License.

\section{REFERENCES}

1. Van der Knaap MS, Valk J. Acute disseminated encephalomyelitis and acute hemorrhagic encephalomyelitis. In: Magnetic Resonance of Myelination and Myelin Disorders, 3rd edition, Springer, New York 2005. p.604.

2. Denes E, Magy L, Pradeau K, et al. Successful treatment of human herpesvirus 6 encephalomyelitis in immunocompetent patient. Emerg Infect Dis 2004;10(4):729.

3. Hart MN, Earle KM. Haemorrhagic and perivenous encephalitis: a clinical-pathological review of 38 cases. J Neurol Neurosurg Psychiatry 1975 Jun;38(6):585-91.

4. Kaji M, Kusuhara T, Ayabe M, et al. Survey of herpes simplex virus infections of the central nervous system, including acute disseminated encephalomyelitis, in the Kyushu and Okinawa regions of Japan. Mult Scler 1996 Sep;2(2):83-7.

5. Silver B, McAvoy K, Mikesell S, et al. Fulminating encephalopathy with perivenular demyelination and vacuolar myelopathy as the initial presentation of human immunodeficiency virus infection. Arch Neurol 1997 May;54(5):647-50.

6. Tsiodras S, Kelesidis T, Kelesidis I, et al. Mycoplasma pneumoniae-associated myelitis: a comprehensive review. Eur J Neurol 2006;13(2):112.

7. Bigi S, Banwell B, Yeh EA. Outcomes after early administration of plasma exchange in pediatric central nervous system inflammatory demyelination. J Child Neurol 2014;30:874-880.

8. Hawkins CP, Mackenzie F, Tofts P, et al. Patterns of bloodbrain barrier breakdown in inflammatory demyelination. Brain 1991 Apr;114(Pt 2):801-10.

9. Zautner AE, Johann C, Strubel A, et al. Eur J Clin Microbiol Inf Dis 2014;33:1019-1027.

10. Keithlin J, Sargeant J, Thomas MK, et al. Systematic review and meta-analysis of the proportion of Campylobacter cases that develop chronic sequelae. BMC Public Health 2014; 14:1203-1222.

11. Levy I, Weissman Y, Sivan Y, et al. Acute encephalopathy associated with campylobacter enteritis. BMJ 1986; 293:424.

12. Orr D, McKendrick MW, Sharrock B. Acute disseminated encephalomyelitis temporally associated with Campylobacter gastroenteritis. JNeurol Neurosurg Psychiatry 2004;75:792-793.

13. Gaig C, Valldeoriola F, Saiz A. Acute disseminated encephalomyelitis associated with Campylobacter jejuni infection and antiganglioside GM1 IgG autoantibodies. J Neurol 2005; 252:613-614.

14. Lepur D, Peterkovic V, Kovacevic SB, et al. Acute encephalopathy associated with Campylobacter jejuni enteritis. Neurol Sci 2012;33:155-158.

15. Yamada A, Miyachi N, Miura T, et al. Long-term poor rapport, lack of spontaneity and passive social withdrawal 
related to acute post-infectious encephalitis: a case report. SpringerPlus 2016;5:345-352.

16. Young JW, Mason DF, Taylor BV. Acute inflammatory encephalomyelitis following Campylobacter enteritis associated with high titre antiganglioside GM1 IgG antibodies. Journal of Clinical Neuroscience 2009;16:597-598.

17. Alicino C, Infante MT, Gnadoglia I, et al. Acute disseminated encephalomyelitis with severe neurological outcomes following virosomal seasonal influenza vaccine. Human Vaccines and Immuno 2014;10:1969-1973.

18. Pillai SC, Hachen Y, Tantsis E, et al. Infectious and autoantibody-associated encephalitis: clinical features and long term outcome. Pediatrics 2015;135:e974-e984.
19. Hahn CD, Miles BS, Macgregor DL, et al. Neurocognitive outcome after acute disseminated encephalomyelitis. Ped Neurol 2003;29:117-123.

20. Shilo S, Michaeli O, Shahar E, et al. Long-term motor cognitive and behavioral outcome of acute disseminated encephalomyelitis. Eur J Ped Neurol 2016;20:361-367.

21. Suppiej A, Cainelli E, Casara G, et al. Long-term neurocognitive outcome and quality of life in pediatric acute disseminated encephalomyelitis. Ped Neurol 2014;50:363-367.

22. Beatty C, Bowler RA, Farooq O, et al. Long-term neurocognitive, psychosocial, and magnetic resonance imaging outcomes in pediatric-onset acute disseminated encephalomyelitis. Ped Neurol 2016;57:64-73. 